\title{
Employees' Emotional Intelligence and Job Performance: A Moderated Mediation Model
}

\author{
Zhang Luyao ${ }^{1, \mathrm{a}}$, Lu Lei ${ }^{1, b^{*}}$ \\ ${ }^{1}$ Department of Business, Macau University of Science and Technology, Taipa, Macau,China \\ a340562427@qq.com \\ b*lucas200846@163.com
}

\begin{abstract}
"Dedication", "patriotism, integrity, and friendliness" have been incorporated into the socialist core values in China as the basic ethical requirements for individuals. Nowadays, predicting employee behaviors and improving employee engagement has been crucial for companies in their human resource management practices. The extent of employee engagement in an enterprise is determined by the care and support perceiving from the organization, which is supported by the Perceived Organizational Support (POS) in social exchange theory. On the basis of emotional theory and social exchange theory, the relationship between emotional intelligence and job performance, as well as the mediating role of employee engagement in this relationship, and the moderating role of organizational support are researched and explored.
\end{abstract}

Keywords: Emotional intelligence, employee engagement, organizational support, job performance

\section{员工的情绪智力与工作绩效: 一个有调节的中介模型 \\ 张鹿瑶 ${ }^{1,}$ a陆否石 ${ }^{1,}$ b*}

\author{
${ }^{1}$ 澳门科技大学商学院，欧仔，澳门，中国 \\ a340562427@qq.com \\ b*lucas200846@163.com
}

\section{摘要}

“敬业”与 “爱国、诚信、友善” 作为对个人的基本道德规范要求，已经被共同纳入到我国社会主义核心价值 观中。当下，企业如何充分预测和了解员工行为，如何提升员工敬业度已经成为企业人力资源管理实践时面临 的实际问题。企业中的员工工作投入程度有赖于他们所感知到的来自组织的关心和支持，这一点已经也被基于 社会交换理论的组织支持感证实。基于情绪理论和社会交换理论，对情绪智力和工作绩效之间的关系, 以及员 工敬业度在此关系中的中介作用和组织支持感的调节作用进行研究和探索。

关键词: 情绪智力, 员工敬业度, 组织支持感, 工作绩效

\section{1. 前言}

随著全球经济一体化, 中国的社会发展日新月异， 在企业竞争不断加剧的今天, 决胜关键就在于拥有一 支高度敬业的员工团队。自 2019 年年末起, 新冠疫 情对于世界经济产生巨大衝击, 经营环境的不确定性 也迫使企业需要员工在巨大的压力下维持稳定的产 出, 以适应变化的市场环境。在过往的研究中, 证实
了员工的情绪智力决定了他们面对压力时可能产生 的情绪以及是否能够正确认知自己的情绪, 并且能够 帮助企业管理者有效预测员工的工作绩效。这对于疫 情下举步维艰的企业实践来说是至关重要的。

随著知识经济时代的到来, 员工的工作性质和组 织实体的变化冲击着员工工作绩效的传统理念。因此, 组织更加关注员工的行为以适应新的挑战 (Griffin et a1.，2007）。在员工层面, 高度的敬业精神能够 
改善员工工作时缺乏注意力和工作积极性不高等问 题，对个体绩效有积极影响。在此背景下，员工敬业 度作为一个组织行为学和人力资源管理领域的新概 念, 是组织行为学方面学者研究的发展趋势。根据 Hewitt（2013）的调查，40\%的被调查员工敬业度不 高, $20 \%$ 的员工处于闲散状态。在全球的组织中, 员 工敬业度依然较低, 对于大多数组织管理者来说, 这 也已经成为需要解决的一个实际问题。

在本研究中的情绪智力, 是指人们感知和有效控 制自身情绪, 并以此指导自身行为, 理解他人并与他 人相处的能力（吕鸿江、韩承轩、王道金，2018）。 Boyatzis（2009）研究表明, 情绪智力能够帮助企业 预测绩效。但对其是以怎样的机制作用于工作绩效, 及发挥该机制所需的条件等问题的探索研究还较少, 而且在探索两者关系的研究中, 对于组织支持感在关 系中的调节效应重视不足。此外, 在以工作绩效作为 被解释变量, 探索情绪智力和员工敬业度的研究中, 对两者的效应分析不够全面。在此背景下, 本研究基 于社会交换理论, 对情绪智力与工作绩效的关系, 以 及员工敬业度在此关系中的中介作用和进行整合性 研究，并探索组织支持感在上述关系中的调节效应。

\section{2. 文献综述}

\section{1. 情绪智力}

情绪智力 ( Emotional Intelligence, EI) 概念 自 1990 年由 Salovey 和 Mayer 首次提出, 作为影响 个体发展的关键概念, 从此在心理学和管理学领域备 受学者的关注。情绪智力本质是社会智力的一部分, 是个人监控自己及他人情绪情感, 并利用这些信息指 导自己行为的能力。根据 Salovey 和 Mayer 的观点, 情绪智力包含以下四个维度: 1 . 自我情感评价, 指一 个人能体会自己的深层次情绪并能够清楚、自然地表 达自身情感的能力（Wong\& Law，2002）；2. 对他人 的情感识别及评价, 指一个人理解和感知周围人情绪 的能力; 3. 情绪控制, 指一个人控制、管理自身情绪, 从心理挫败中迅速恢复的能力; 4 . 情绪利用, 指一个 人通过运用情绪推动工作表现和向建设性行为发展 的能力 (Joseph\& Newman, 2010)。本文基于相关文 献, 定义情绪智力为个体评估、表达、认知和使用自 己及他人情绪的能力。

大量研究支持情绪智力对于个人和组织的绩效 有显著的影响, Wong\& Law (2002) 和 Stein and Book (2010) 等的研究证实, 员工的情绪智力越高越 容易获得职业成功, 情绪智力与其工作绩效之间存在 显著的正向关系。

\section{2. 员工敬业度}

员工敬业度是指组织成员对于工作的自我角色 的管理, 以及在工作角色中对自身精力认知和情感的 表达运用（Kahn, 1990）。员工敬业度包括三个心理
条件: 1. 心裡意义, 可以被视为一种感觉, 是员工对 投资于身体、认知或情感的能量的当前回报，缺乏则 意味著人们得到较少的工作绩效; 2 . 心理安全感, 即 一种主观感受, 是个体在组织中定位的三种重要心理 状态之一, 能够让个体充分展现自我, 而无需担心其 会对个人地位、自我形象或职业生涯产生负面影响; 当情况是不可预知或不一致, 以及存在威胁时, 员工 敬业度将会是不高或不安全的; 3 . 可用性, 指在某个 特定的时刻, 对员工在身体、情绪或心理上有意义。

过往的研究发现, 工作中的六个方面和员工敬业 度相关: 可持续的工作量、适当的表彰和奖励、选择 和控制、社会公平和争议、支持性的工作感受, 以及 有意义和有价值的工作 (Maslach, 2001)。以往研究 主要将员工敬业度视为对工作敬业, 而员工敬业度还 取决于在具体情境的角色, 因而, 可以分为对工作敬 业和对组织敬业（Saks，2006）。

\section{3. 工作绩效}

工作绩效（Job Performance）是指对于特定目 标的实现程度, 可以分为个体、团体和组织层面, 而 不同层面对于工作绩效概念的界定也存在差异, 本文 主要探究个体层面的工作绩效, 回溯国内外有关工作 绩效的研究可以将相关研究总结为三个方面:

1. 以结果为导向的工作绩效, 李宝元 (2002) 以 结果为导向将工作绩效界定为员工在生产效率、生产 质量和服务质量等方面的表现和成绩。2. 以行为为导 向的工作绩效, 被定义为员工做出的所有对组织目标 有贡献的行为。3. 综合工作绩效, Chiang \& Hsieh （2012）认为工作绩效应该包括员工的工作结果与实 现该结果的过程, 即员工的行为与结果以及能力、态 度、工作方式等等。

员工个体工作绩效的影响因素研究一直是心理 学和管理学研究的重要话题, 探索工作绩效的影响因 素, 对提升员工的工作积极性具有重要意义。

\section{4. 组织支持感}

Eisenberger 等人 (1986) 提出了组织支持感 (Perceived Organizational Support, POS) 的概 念, 认为是员工对于组织重视其贡献和关注其幸福感 的全面看法, 包括: 1. 员工对组织是否重视其贡献的 感受; 2 . 员工对组织是否关注其幸福感的感受。

组织支持感的定义为, 员工感知到的组织对他们 工作上的支持、对他们利益的关心和对他们价值的认 同。基于互惠原则, 组织在资源和情感上给予员工更 多的支持, 感受到高度的组织支持感的员工会产生关 心组织利益的义务感、归属感和情感需求的满足感, 增加对组织的付出回报。

过往的研究表明, 组织支持感与各种重要的组织 成果相关。Rhoades \& Eisenberger (2002) 的研究 
表明组织支持感和退缩行为（如旷工、迟到和离职） 呈负向关系。

\section{3. 研究假设推理}

\section{1. 情绪智力与工作绩效}

情绪智力是不同于传统智力和人格特质的一种 独立智力形式, 体现了个体为驾驭自己和他人的情感 情绪, 区分它们之间的差异, 并能使用这些指导自己 思考和行动的能力 (Joseph et al. , 2015; 张辉华, 2014）。自从情绪智力的概念提出以来, 组织行为学 领域的相关研究从情绪自我评估、他人情绪评估、情 绪使用和情绪调控几个方面解释情绪智力对于员工 行动的作用机制。情绪智力的高低会影响道个体对周 围环境的感知, 作用于个体行为和员工行为效果 (王 仙雅等, 2013)。依据情绪智力理论, 拥有良好情绪 智力的员工不仅能够在组织中合理运用特定的情绪 技能, 更重要的是根据情境的变化能够调节自身的情 绪状态, 与同事有效沟通, 表现出积极的工作态度。 事实证明, 情绪智力对工作绩效具有较强的预测能力

(Lopes et al.,2006; Offerman et al., 2004)。 文献分析发现, 高情绪智力的员工能够更好控制自己 情绪, 易于表现强烈的合作意识, 营造出融洽的交流 沟通氛围, 建立更好的人际关系, 从而取得更好的绩 效。由此推测:

$H 1$ : 情绪智力对员工工作绩效具有正向影响作用。

\section{2. 情绪智力与员工敬业度}

敬业度对员工有著积极的影响, 它在激励员工实 现其个人和组织目标方面发挥著关键作用 (Judge et al., 2002)。恰当的情绪和感受对组织与个人 都具有战略价值和影响。过去的许多研究已经表明, 情绪智力被视为积极工作态度的重要预测因素。情绪 智力较高的员工拥有更高的工作满意度, 因为他们能 够认知与调节自己的情绪。（Sy et al., 2006) 在 另一项对于印度专业人士的研究中, Swatee \& Aakanksha（2015）发现他们的情绪智力与敬业度之 间存在积极的关系, 因为情绪智力较高的人能够表现 出更多的活力、奉献精神和专注度, 而情绪混乱的员 工被认为会降低其个人的幸福感, 导致工作懈急的现 象。高情绪智力的人被认为在用有趣而有力的方式在 工作中表达自己的想法, 从而对组织和个人产生积极 的影响。由此推测:

$\mathrm{H} 2$ ：情绪智力对员工敬业度具有正向影响作用。

\section{3. 员工敬业度与工作绩效}

工作绩效是指对于特定目标的实现程度, 可以分 为三个层面, 本文主要讨论个体层面的工作绩效, 即 员工个体在工作过程中所有与组织目标实现有关的 行为与结果的综合。本文跟据学术界重广泛界定的三
种绩效观中的综合工作绩效, 将工作绩效划分为任务 绩效和关系绩效两个维度。

员工的敬业度包括认知敬业、情感敬业和行为敬 业。基于社会交换理论, 认知敬业度高的员工能够敏 锐感知对组织的认可, 产生提高自身工作绩效为组织 目标贡献的动机（Schaufeli and Bakker，2004）。 员工的情感敬业会影响到员工的工作满意度, 促使员 工实现更多组织目标的倾向, 对关系绩效有积极影响 （陈明淑、申海鹏，2015）。行为敬业会影响员工的 工作投入水平, 刺激工作能力的提升, 对员工的事业 发展和任务绩效有积极影响(王默凡、孙敏健, 2016)。 基于以上，提出假设如下：

H3: 员工敬业度对工作绩效有正向影响。

\section{4. 组织支持感对情绪智力与员工敬业度和 工作绩效关系的调节}

前文的分析指出, 基于互惠原则, 高情绪智力的 员工对组织的激励与组织积极的氛围的感知更为敏 感, 高度的组织支持感的感知有助于员工做出有利组 织的行为, 有更多的工作投入和绩效表现, 更少的逃 避行为 (Rhoades \& Eisenberger, 2002)。相反, 如 果对组织的支持感感知较少, 员工会有更少的组织义 务感和对组织的归属感。因此, 本文提出

$\mathrm{H} 4$ : 组织支持感在情绪智力与员工敬业度的关系 中具有调节作用: 与较高的组织支持感相比, 员工组 织支持感较低时情绪智力对员工敬业度的负向影响 更强。

$\mathrm{H} 5$ : 组织支持感在员工敬业度与员工工作绩效的 关系中具有调节作用: 与较高的组织支持感相比, 员 工组织支持感较低时员工敬业度对员工工作绩效的 负向影响更强。

\section{5. 研究模型}

综上，基于对情绪智力，员工敬业度，工作绩效 和组织支持感四个变量的文献整理和分析, 建立了如 下研究模型。 


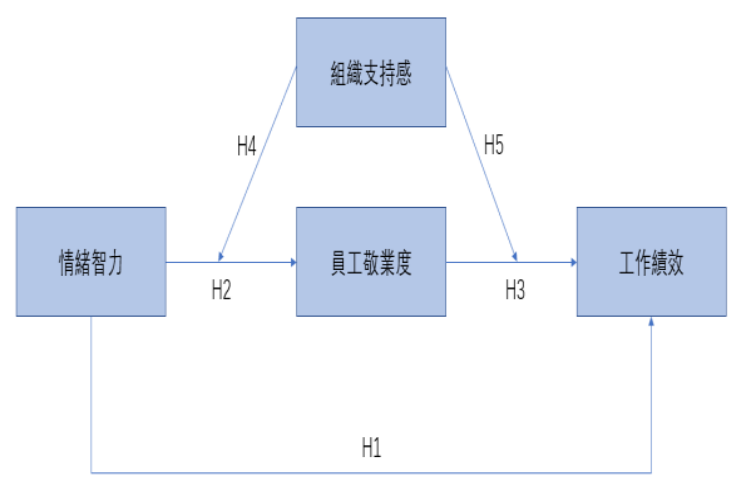

图 1 研究模型图

\section{4. 研究意义}

\section{1. 理论意义}

情绪智力的概念自 1990 年被提出后, 近年已成 为组织心理学的研究热点。现有的研究主要基于情绪 智力与员工工作绩效之间关系的研究, 普遍忽略了对 中介机制和调节影响因素的深入探讨。回溯过往对于 情绪智力的研究, 国外多数文献是围绕领导行为、社 会网络和认知能力等理论, 探索情绪智力和员工个人 绩效之间的关系。国内相关的研究 (张辉华、黄婷婷, 2015; 唐文清、香梅、张敏强等, 2015）主要是基于 学生群体和企业工作者展开的, 但是例如情绪智力是 通过怎样的内在机制影响个体绩效和其发挥作用的 边界等问题仍有待深入探索, 并且这方面的研究也缺 乏有力的理论解释。总体而言, 情绪智力相关的众多 研究问题未达成一致, 其在心理学和管理学领域的实 践探索仍处在初步发展阶段。针对现存的研究缺口, 本文基于情绪理论和社会交换理论,构建了包括中介 环节的调节效应模型概念, 对现有的情绪智力和工作 绩效相关理论进行了补充的探索研究。

\section{2. 实际意义}

从事服务业工作的员工是通过提供服务与被服 务者建立关系的, 相关研究表明, 在包含大量人际交 往和较高情绪互动需求的工作中, 情绪智力对员工的 工作绩效具有较强预测作用 (Maslach et al., 2001）。

首先, 本文以情绪智力作为解释变量, 探索员 工敬业度的前因和提升机制以及组织支持感的效果 研究, 研究结果对企业管理实践具有重要意义, 对如 何塑造员工忠诚感和提升工作积极性具有借鑑意义。

其次, 本文以员工工作绩效作为被解释变量, 考 察员工敬业度的积极效应, 研究结论的验证有助于引
起管理实践中管理者对员工敬业度的重视, 关注员工 绩效的提升, 从而推动企业健康持续的成长。

\section{5. 结论}

本研究基于社会交换理论, 对情绪智力与工作绩 效的关系, 以及员工敬业度在此关系中的中介作用和 进行整合性研究, 并探索组织支持感在其中的调节效 应。

研究采用问卷调查方式, 以获取可靠和现实的一 手研究数据, 并对问卷数据进行共同方法性偏差进行 评估, 利用结构方程模型进行因素分析来检验研究的 变量及模型。除了不可避免的同源偏差问题, 因为受 到其他客观因素影响, 以及组织限制问题, 研究必然 存在一些局限和不足: 研究样本的选取、测量工具的 局限、测量量表的选择等等。

\section{REFERENCES}

[1] Aon Howitt Ltd. 2013. Trends in Global Employee Engagement Report [R]. London : Aon Hewitt Ltd.

[2] Boyatzis R.E. 2009. Competencies as A Behavioral Approach to Emotional Intelligence[J]. Journal of Management development, 28 (9):749-770.

[3] Chiang C.F., Hsieh T.S. 2012. The Impacts of Perceived Organizational Support and Psychological Empowerment on Job Performance: The Mediating Effects of Organizational Citizenship Behavior [J]. International Journal of Hospitality Management, 31 (1): 180-190.

[4] Griffin M.A., Neal A., Parker S.K. 2007. A New Model of Work Role Performance: Positive Behavior in Uncertain and Interdependent Contexts[J]. Academy of Management Journal, 50 (2):327-347.

[5] Joseph D L, Jin J. Newman D A. et al. 2015. Why Does Self-Reported Emotional Intelligence Predict Job Performance? A Meta-Analytic Investigation of Mixed Ei[J]. The Journal of applied psychology, 100 (2): 298-342.

[6] Joseph D.L. \& Newman D.A. 2010. Emotional Intelligence: An Integrative Meta-Analysis and Cascading Model [J]. Journal of Applied Psychology, 95 (1): 54-78.

[7] Judge T.A., Bono, J. E., Ilies, R., et al. 2002. Personality and Leadership: A Qualitative and Quantitative review [J]. Journal of Applied Psychology, 87 (4), 765.

[8] Kahn W.A. 1990. Psychological Conditions of Personal Engagement and Disengagement at Work [J]. Academy of Management Journal, 33 (4): 692724. 
[9] Lopes P.N., Grewal D., Kadis J., et al. 2006. Evidence that Emotional Intelligence is Related to Job Performance and Affect and Attitudes at Work [J]. Psicothema, 18 (1): 132-138.

[10] Maslach C., Schaufeli W.B., Leiter M.P. 2001. Job Burnout [J]. Annual Review of Psychology, 52 (3): 397-422.

[11] Ng K.M., Wang C., Zalaquett C.P., et al. 2007. A Confirmatory Factor Analysis of the Wong and Law Emotional Intelligence Scale in A Sample of International College Students[J]. International Journal for the Advancement of Counselling, 29 (34): 173-185.

[12] Offerman L.R., Bailey J.R., Vasilopoulos N.L., et al. 2004. The Relative Contribution of Emotional Competence and Cognitive Ability to Individual and Team Performance [J]. Human Performance, 17 (2): 219-243.

[13] Rhoades L, Eisenberger R. 2002. Perceived Organizational Support: A Review of the Literature[J]. Journal of Applied Psychology,87 (3): 698-714.

[14] Saks A.M. 2006. Antecedents and Consequences of Employee Engagement [J]. Journal of Managerial Psychology, 21 (7): 600-619.

[15] Salovey P., D Mayer J. 1990. Emotional Intelligence $[\mathrm{J}]$. Imagination, Cognition-andpersonality, (9): 185-211.

[16] Schaufeli W.B., Bakker A.B. 2004. Job Demands, Job Resources, and Their Relationship with Burnout and Engagement: A Multi Sample Study [J]. Journal of Organizational Behavior, (25): 293-315.

[17] Stein S.J., Book H. 2013. The EQ edge: Emotional intelligence and your success [M]. Psychology Press.

[18] Swatee S., Aakanksha V. 2015. Role of Emotional Intelligence on Employee Engagement: A Study among Indian Professionals [J]. International Journal of Business and Management, 10 (6): 224233.

[19] Sy T., Tram S., O'hara, L.A. 2006. Relation of Employee and Manager Emotional Intelligence to Job Satisfaction and Performance [J]. Journal of Vocational Behavior, 68 (3), 461-473.

[20] Tsui A. 1997. Alternative Approaches to the Employee-Organization Relationship: Does Inducement in Employees Pay Off? [J]. Academy of Management Journal, 40 (5): 1089-1121.
[21] Wong C.S. \& Law K.S. 2002. The Effects of Leader and Follower Emotional Intelligence on Performance and Attitude: An Exploratory Study [J]. The leadership quarterly, 13 (3): 243-274.

[22] Chen M.S. \& Sheng H.P. 2015. An empirical study on the relationship between trust, engagement and job performance in an organization [J]. Journal of Financial Theory and Practices, 36 (3): 113-118.

[23] Huang L. \& Chen W.Z. 2013. The influence of two interpersonal orientations on subordinates' job performance - Mediating role of the quality of leader-member exchange [J]. Management Review, 27(5):178-187.

[24] Li B.Y. 2002. Strategic incentive _- Essence of human resource management in modern enterprises [M]. Beijing: Economic Science Press.

[25] Lv H.J. et al., 2018. The Relationship Between Emotional Intelligence and Leadership Effectiveness: A Meta-Analysis [J]. Advances in Psychological Science, 26 (2): 204-220.

[26] Tang W.Q. et al., 2015. The influence mechanism of emotional intelligence and moving interpersonal network on adaptability of college students: The multilayer mediating effect model [J]. Psychological science, 38 (3): 618-624.

[27] Wang M.F. \& Sun J.M. 2016. Research on the mechanism of employee vocation orientation on job performance — Mediating role of employee engagement [J]. Soft science, 30 (5): 100-103.

[28] Wang X.Y. et al., 2013. Research on the influence mechanism of research pressure on research performance — The Moderating Effect of Academic Atmosphere and Emotional Intelligence [J]. Research of Science, 10 (10): 1564-1571.

[29] Zhang H.H. \& Huang T.T. 2015. The effect mechanism of emotional intelligence on performance: The continuous mediators of team trust perception and friend network center [J]. Nankai Management Theory, 18 (3): 141-150.

[30] Zhang H.H. 2014. Individual Emotional Intelligence and Task Performance: Perspective of Social Networking [J]. Journal of psychology, 46 (11): 1691-1703.

[31] Zhang X. \& Huang Y.F. 2015. Research on the relationship between role clarity, employee engagement and extra-role behavior [J]. Human Resource Management, (12): 102-106. 